\title{
CONSTRUCTIVE QUANTUM FIELD THEORY
}

\author{
ARTHUR JAFFE \\ Harvard University, Cambridge, MA 02138, USA \\ E-mail: jaffe@physics.harvard.edu \\ Web: www.arthurjaffe.com
}

\begin{abstract}
We review the emergence of constructive quantum field theory, we discuss how it fits into the framework of mathematics and physics, and we point to a major unsolved question.
\end{abstract}

\section{Background}

The pioneering work of early non-relativistic quantum theory led to the understanding that quantum dynamics on Hilbert space is a comprehensive predictive framework for microscopic phenomena. From the Bohr atom, through the nonrelativistic quantum theory of Schrödinger and Heisenberg, and the relativistic Dirac equation for hydrogen, agreement between calculation and experiment improved rapidly over time. The incorporation of special relativity and field theory into quantum theory extended the scope of perturbative calculations, and these were tested through precision measurements of spectra and magnetic moments. Beginning in the 1940's, experimental tests of the Lamb shift and the anomalous magnetic moment of the electron detected effects that one can ascribe to fluctuations in quantum electrodynamics. These effects deviated numerically from the predictions arising from equations that describe a fixed number of particles, so they were accurate tests of the quantum field hypothesis. Today these experiments have evolved to yield quantitative agreement with the most precise observations and calculations achieved in physics. For example, the anomalous magnetic moment of the electron is known theoretically and experimentally to amazing precision: $(g-2) / 2=0.001159652200( \pm 40)$. The success of this work, as well as the success of other less accurate, but compelling, predictions for weak and strong interactions, convince us to accept quantum field theory as the correct physical arena to describe particle physics down to the Planck scale.

But the success of relativistic field theory calculations and of perturbative renormalization also led to a logical puzzle: is there any physically-relevant, relativistic quantum field theory that is also mathematically consistent? Put differently, can one give a mathematically complete example of any non-linear theory, relevant for the description of interacting particles, whose solutions incorporate relativistic covariance, positive energy, and causality? One must understand perturbative renormalization in order to resolve this problem, and have control over renormalization from a non-perturbative (or "exact") point of view. In fact, one needs to overcome sophisticated problems, such as whether a field theory may appear correct on a perturbative level, while it may have no meaning at a non-perturbative level. Doubts about quantum electrodynamics or scalar meson theory were raised early by Dyson and Landau. They recur from the point of view of the renormalization group in the work of Kadanoff and Wilson, as well as in the analysis of "asymptotic 
freedom" in the 1970's. In four dimensions, this has focused attention on finding a solution to a non-abelian Yang-Mills theory.

Assuming a positive answer to this existence question, then can one develop a calculational scheme to determine properties of such an example, both perturbatively and non-perturbatively? Strong-coupling calculations, as well as calculations near critical values of the coupling constants, have been the most elusive to understand. Thus one wants to understand both the quantitative structure of field theories, as well as qualitative features such as the dependence of the theories as functions on the space of coupling constant parameters.

At the same time, physicists have tried to make further theoretical progress through ambitious attempts to imbed quantum field theory within a theory of strings, by which they hope to combine quantum theory with general relativity, and to predict the structure of space-time. There is also the appealing attempt to integrate non-commutative geometry into the picture. One would like to introduce the notion of quantization directly at the level of space-time, and to describe field theories on quantum space-time, rather than applying quantization to fields that live on a classical space-time. For the time being, all these methods remain beyond the realm of full understanding.

\section{The Emergence of CQFT}

Constructive quantum field theory (CQFT) was formulated forty years ago as an effort to find specific examples of non-linear quantum fields that fit within a mathematically complete description of quantum mechanics. Prior to the constructive field theory program, only a few exactly soluble relativistic field theories existed. Either they were free fields, or else they had solutions that could be expressed as functions of free fields (such as the Schwinger and Thirring models). In either case, they described (or appear to describe) particles without interaction or scattering.

The emergence of constructive quantum field theory led to the direct attack on showing that solutions exist to the variational equations arising from particular Lagrangians. Constructive field theorists took perturbation theory as a reliable guide to the behavior of a particular equation. One could incorporate this information into the mathematical analysis, leading to the proof of exact results based on perturbative guidance.

The most basic questions raised by CQFT in the 1960's revolved about whether examples of quantum field theories exist within the axiomatic frameworks formulated by Wightman or by Haag and Kastler. The Wightman axioms describe the vacuum expectation values of products of fields that transform covariantly under the action of the group of inhomogeneous Lorentz transformations $\{a, \Lambda\}$, where $\Lambda$ designates a homogeneous Lorentz transformation and where $a$ denotes a space-time translation. Thus they involve constructing the fields $\varphi(x, t)$, along with a unitary, positive-energy representation $U(a, \Lambda)$ of the inhomogeneous Lorentz group, both of which act on the Hilbert space $\mathcal{H}$ of the quantum theory. Furthermore, one requires that a zero-energy ground state $\Omega \in \mathcal{H}$ of the Hamiltonian dynamics exists, called the vacuum. This vector $\Omega$ should remain invariant under the full group $U(a, \Lambda)$, and Wightman assumes furthermore that the vacuum is unique. In other 
words, zero is a simple eigenvalue of $H$.

The unitary representation $U(a, \Lambda)$ of the Lorentz group of space-time symmetries determines a $*$-automorphism group of transformations of fields,

$$
\sigma_{a, \Lambda}: \varphi \rightarrow U(a, \Lambda) \varphi U(a, \Lambda)^{*}
$$

Scalar boson fields are characterized by the transformation property

$$
\left(\sigma_{a, \Lambda} \varphi\right)(\hat{x})=\varphi(\Lambda \hat{x}+a)
$$

where $\hat{x}=(x, t)$ denotes a space-time point, and spinor fields or vector fields have their own transformation laws. The Haag-Kastler axioms deal with the fields (or bounded functions of the fields), along with a positive energy representation of the automorphism group $\sigma_{a, \Lambda}$. One advantage of dealing with the automorphisms $\sigma_{a, \Lambda}$, rather than with $U(a, \Lambda)$, is that one can construct the automorphism group locally, using different generators in different bounded space-time regions, and then patch together a global automorphism group from the local pieces. This procedure may therefore avoid problems with constructing the global generators of $U(a, \Lambda)$.

Even this more general framework lacked examples. One hoped that a real breakthrough on the side of analysis would ultimately lead to an understanding of all alternative axiom schemes. So one attempted a frontal attack on various existence problems, both on Minkowski space, and also on variations of the frameworks appropriate to compactified configuration space. At the inception of these CQFT efforts, even finding examples of super-renormalizable Lagrangians in twodimensional space-times appeared extremely difficult, if not beyond reach. Wightman presented an optimistic point of view in 1964 at Cargèse ${ }^{1}$ mainly from the point of view of understanding the vacuum expectations of a number of known soluble examples. This author gave a Hamiltonian framework of estimates to construct regularized fields, ${ }^{2}$ and Lanford gave another. ${ }^{3}$

Still examples without ultra-violet regularization eluded researchers. Kurt Symanzik laid out a ground breaking framework to study the analytic continuation (to imaginary times) of vacuum expectations. ${ }^{4}$ However, some years later Symanzik evaluated his own functional integral program as lacking, when he compared it point by point with the Hamiltonian approach. ${ }^{5}$ At the time Symanzik's methods appeared beyond the scope of possible mathematical analysis, and he decided to pursue other problems.

\section{The First Examples}

Over the following twelve years, 1965-1976, constructive field theorists finally achieved the non-perturbative construction of field theories with non-linear interaction in two-dimensional and in three-dimensional space-times. Both the Hamiltonian approach and the functional integral approach ultimately proved successful. In fact, using features of both methods turned out to be the most powerful point of view. Looked at in perspective, it is a shame that Symanzik abandoned his approach when he did.

Early progress on answering these questions in a two-dimensional space-time owed a great deal to the 1965 paper of Nelson ${ }^{6}$ showing stability for the $\varphi^{4}$ Hamiltonian of a field $\varphi(x, t)$ defined on a cylindrical space-time, $x \in S^{1}, t \in \mathbb{R}$. The 
stability estimate leads via the Friedrichs extension to a self-adjoint extension of $H$, but does not in itself give uniqueness of that extension (essential self-adjointness), and its concomitant dynamics $\varphi(x, t)=e^{i t H} \varphi(x, 0) e^{-i t H}$.

The proof of essential self adjointness, as well as the first non-trivial example of solutions to the relativistic field equation in a 2-dimensional Minkowski spacetime $(x, t) \in \mathbb{M}^{2}$ appeared in a series of papers written by this author jointly with Glimm. ${ }^{7,8,9,10}$ This hyperbolic, non-linear equation for $\varphi(x, t)$ has the form

$$
\square \varphi+\varphi+\lambda \varphi^{3}=0
$$

where $\square$ denotes the wave operator $\square \varphi=\varphi_{t t}-\varphi_{x x}$, where $\lambda$ is a positive parameter, and where the non-linear power $\varphi^{3}$ in the equation requires special definition. The solution to this equation locally is not an operator valued field; rather it is an operator-valued distribution. As the equation (3) is non-linear, its mathematical analysis requires an intermediate step in which one studies a smoothed out (or regularized) equation. In this case, the regularization can be achieved by truncating the Fourier representation of the field, $\tilde{\varphi}(k)=\int \varphi(x, 0) e^{i k x} d x$, when the magnitude of the Fourier variable $k$ exceeds $\kappa$. The equation (3) is obtained in the limit $\kappa \rightarrow \infty$.

The equation (3) is known as the $\varphi_{2}^{4}$ wave equation, where the subscript denotes the dimension of space-time, and the quartic power signifies that the interaction energy density $\frac{1}{4} \lambda \varphi^{4}$ is quartic. The methods leading to these results were soon generalized to yield the solution to equations on $\mathbb{M}^{2}$ with a large class of polynomial non-linearities with energy bounded from below, also known as the $\mathcal{P}(\varphi)_{2}$ equations. Extensive references can be found in Quantum Physics. ${ }^{11}$

The initial data $\varphi, \varphi_{t}$ for the partial differential equation (3) are operator-valued, rather than the usual distributions that one encounters in non-linear PDE. The initial data are required to satisfy the usual canonical commutator constraints of quantum theory,

$$
\left[\varphi(x, 0), \varphi_{t}\left(x^{\prime}, 0\right)\right]=i \delta\left(x-x^{\prime}\right) I .
$$

Here $\delta$ denotes the Dirac measure. In addition one requires

$$
\left[\varphi(x, 0), \varphi\left(x^{\prime}, 0\right)\right]=\left[\varphi_{t}(x, 0), \varphi_{t}\left(x^{\prime}, 0\right)\right]=0 .
$$

The presence of the singularities in (4) clearly indicate the singularity of the solutions $\varphi$, and signals that the non-linear power $\varphi^{3}$ that occurs in $(3)$ requires a special definition; this is a simple example of the phenomenon of "renormalization." In this case the "cube" is replaced by a cubic polynomial $\varphi^{3}-3 c \varphi$, where $c$ is a divergent constant, and the coefficient 3 is conventional. This constant is suggested by lowest order perturbation theory. Let us consider a sequence of regularized equations as introduced above, parameterized by $\kappa$, and with non-linearity $\varphi_{\kappa}^{3}-3 c_{\kappa} \varphi_{\kappa}$. This non-linearity converges as $\kappa \rightarrow \infty$ and $c_{\kappa} \sim \ln \kappa$, if we choose the coefficient of the logarithm according to lowest order perturbation theory. Thus the individual terms in

$$
\varphi^{3}=\lim _{\kappa \rightarrow \infty}\left(\varphi_{\kappa}^{3}-3 c_{\kappa} \varphi_{\kappa}\right)
$$


have no meaning, but their sum is a defined by a well-behaved limit and provides the non-linearity in the wave equation (3). The root of the difficulty in establishing stability stems from the fact that the "normal-ordered" interaction energy, integrated over a compact domain $I$,

$$
V_{I}=\lim _{\kappa \rightarrow \infty} \frac{1}{4} \int_{I}\left(\varphi_{\kappa}^{4}-6 c_{\kappa} \varphi_{\kappa}^{2}+3 c_{\kappa}^{2}\right) d x=\frac{1}{4} \int_{I}: \varphi^{4}: d x
$$

is a densely defined operator, but it is unbounded from below. Here the colons $: \cdot$ : denote substituting a Hermite polynomial for each monomial of $\varphi$, also called normal ordering. Stability is the statement that the operator $H(I)=H_{0}+V_{I}$ is bounded from below, where $H_{0}$ is the Hamiltonian for the linear wave equation $\square \varphi+\varphi=0$, with the zero-energy ground state given by the Fock no-particle state $\Omega_{0}$. In fact, $c_{\kappa}=\left\langle\Omega_{0}, \varphi_{\kappa}^{2}(x, 0) \Omega_{0}\right\rangle$. In the case that $V_{I}$ is defined in $(7)$, the sum $H(I)$ happens also to be densely defined.

The original construction exhibited that the domain of dependence of the fields has the expected unit velocity of propagation. The Lorentz boost can be locally implemented by the first moment of the energy density. By constructing a selfadjoint, local generator of the Lorentz boosts, we showed that the local algebras of observables constructed from the field form a Lorentz-covariant net, and therefore the field theory has the desired Haag-Kastler properties. Therefore, the quantum theory associated with the solution to the equation (3) gave the first non-trivial example of the Haag-Kastler axiom scheme. ${ }^{12}$

\section{Quantum Theory as Statistical Physics}

The introduction by Schwinger of Euclidean quantum field theory, and the realization by Kurt Symanzik that Euclidean fields have beautiful, Euclidean-covariant functional integral representations, ${ }^{4}$ led to a fascination with Euclidean phenomena. These functional integral representations yield a Feynman-Kac representation of the heat kernel $e^{-t H}$. They also have the interpretation of being a statistical mechanics average of classical fields (over a configuration space of Euclidean field configurations), weighted by the Boltzmann probability $e^{-S_{E}}$. Here $S_{E}$ denotes the classical action functional continued to Euclidean (imaginary) time. The Euclidean $n$-point Green's functions are the $n^{\text {th }}$-moments of the measure. Furthermore, Symanzik showed that a $\varphi^{4}$ interaction corresponded to the intersection of two random paths. The problem was that there was no definition of the putative functional integral as a true integral, given by a countably-additive Borel measure.

Quite aside from the validity of a functional-integral representation for the Euclidean Green's functions, there was another mystery. The existence of Euclidean Green's functions (the analytic continuation of vacuum expectation values to purely imaginary times) follows from the positive energy assumption of the Wightman axioms. However, one might begin by assuming the existence of a set of Euclidean Green's functions, without knowing a priori that these Green's functions arose from a field theory. Could one then find sufficient conditions to justify the analytic continuation back to a quantum theory on a Hilbert space? In other words, could one base a construction of field theories on establishing the existence of the Euclidean Green's functions? 
In 1971, Nelson introduced a probabilistic solution to this problem, ${ }^{13}$ based on the construction of a Markov field, motivated by Symanzik. Nelson's mathematically complete construction has as a consequence the existence of a Hilbert space and a quantum field acting on that space. This method assumes an a priori Markovian structure that can easily be verified for the free Euclidean field, and thus gave rise to the free Markov field. However, this structure is special to bosons, and it presented difficulty in verifying the Markov hypothesis for interacting fields. The Markov framework is sufficient, but it does not give an equivalence of Euclidean and Hilbert space methods. It was used in very interesting ways by Guerra, Rosen, and Simon. ${ }^{14,15}$

Osterwalder and Schrader discovered another solution based on properties of the Euclidean Green's functions. ${ }^{16}$ They showed that their axioms for Euclidean Green's functions are equivalent to the Wightman theory on Minkowski space. Following this discovery, Euclidean fields became the fundamental tool to investigate Minkowski field theory. The beautiful simplicity of this approach is that it relies on a positivity condition, and in many cases this positivity is easy to preserve in approximating (cutoff) field theories. Their condition of reflection (or Osterwalder-Schrader) positivity yields the existence of the Hilbert space $\mathcal{H}$, along with the existence of an positive-energy Hamiltonian, and an analytic continuation from Euclidean space to Minkowski space. In the case of a free bosonic field with two-point Euclidean Green's function $C$, with $P_{+}$the orthogonal projection in $L^{2}$ onto $t \geq 0$, and with $\Theta$ the reflection in the $t=0$ plane, the Osterwalder-Schrader positivity condition states

$$
0 \leq P_{+} \Theta C P_{+}
$$

It turns out ${ }^{17}$ that this positivity is a consequence of the classical operator monotonicity condition for Green's functions of the Laplacian on $P_{+} L^{2}\left(\mathbb{R}^{d}\right)$,

$$
C_{D} \leq C \leq C_{N}, \quad \text { where } \quad C=\left(-\Delta+m^{2}\right)^{-1},
$$

and where $C_{D}$ (respectively $C_{N}$ ) represents the Green's function of $-\Delta+m^{2}$ with Dirichlet (respectively Neumann) data on the $t=0$ plane. The reflection positivity condition also can be established for interacting scalar, fermion, or gauge fields with local interactions. Since positivity is preserved under limits, appropriate convergence of Euclidean Green's functions yields positivity after removal of the cutoffs.

The Euclidean methods lead to mathematically-sound, functional-integral representations of the solutions to field theory problems. These representations often reflect underlying symmetries of the field theories. The Euclidean methods also apply to theories with fermions, at least for examples with interactions that are quadratic in the fermions. ${ }^{18}$ This is the case for free and for "Yukawa type" interactions, used extensively in physics.

These methods have been realized in the two-dimensional and three-dimensional examples. The explicit integral representations lend themselves to the nonperturbative analysis of the examples. Basically two methods were developed to analyze these functional integrals. The correlation inequality method ${ }^{15,11}$ was suitable for a certain class of bosonic Lagrangians. It is based on the fact that in these 
examples the Euclidean Green's functions are positive and monotonic in the volume, as is often the case in bosonic classical statistical mechanics. The expansion $\operatorname{method}^{19,20,11}$ is based on developing convergent expansions in the coupling constant $\lambda$. This method applies for coupling constants $0<\lambda$ sufficiently small. These expansions are not power series (which are known to diverge); however the $n^{\text {th }}$-term in these expansions is $O\left(\lambda^{n}\right)$ as $\lambda \rightarrow 0$. The $n^{\text {th }}$-term has the form $\lambda^{n} G_{n}(\lambda)$, where $\left|G_{n}(\lambda)\right| \leq O(1)$ as $\lambda \rightarrow 0$. These expansion methods are not limited to bosonic Lagrangians, but they are limited to the coupling constant $\lambda$ being small. The expansion methods, when applicable, ultimately yield greater qualitative control over the solutions. In some cases both methods can be used, a great advantage. We return to these two methods in the following section.

\section{The Wightman Axioms and a Mass Gap}

The Osterwalder-Schrader construction shows that if a Euclidean functional integral satisfies reflection positivity and certain bounds on moments, then it yields a relativistic field theory. Furthermore spectral properties of the Hamiltonian and the momentum operators in the resulting field theory can be deduced from cluster properties of moments of the measure that determines the functional integral. Thus the Euclidean functional integrals became a natural and powerful tool for the study of the details of the spectrum of the Hamiltonian.

We assume that the Hamiltonian $H$ is positive, $0 \leq H$, and furthermore that it has the normalized eigenvector $\Omega$ with eigenvalue 0 , namely $H \Omega=0$. The Hamiltonian $0 \leq H$ is defined to have a mass gap, if in addition $H$ has no spectrum in some interval $(0, m)$, where $m>0$. The physical consequence of the existence of a mass gap is that the lightest particle described by the Hamiltonian $H$ has a mass greater or equal to $m$. Assuming that the Hamiltonian $H$ and the momentum operator $P$ commute, we can define the mass operator as the positive square root $M=\sqrt{H^{2}-P^{2}}$. The mass operator labels Lorentz-invariant hyperboloids in the energy-momentum spectrum, and in a covariant theory, a mass gap $(0, m)$ for $H$ means there also exists a mass gap $(0, m)$ in the spectrum of $M$.

Consider the situation where the unit vector $\Omega$ is a null vector (ground state) of $H$, and any vector $\chi \in \mathcal{H}$ satisfies the bound

$$
\left.\left|\left\langle\chi, e^{-t H} \chi\right\rangle-\right|\langle\Omega, \chi\rangle\right|^{2} \mid \leq\|\chi\|^{2} e^{-m t},
$$

for all $t>0$. This situation is equivalent to the statement that 0 is a simple eigenvalue of $H$ with eigenvector $\Omega$, and that $H$ has a mass gap of magnitude $m$. In fact, we would like a sufficient condition on expectations to ensure the existence of a mass gap. One needs only to show that there exists a densely defined functional $f(\chi)$ on $\mathcal{H}$ with domain $\mathcal{D}(f) \ni \Omega$, such that

$$
\left.\left.\left|\left\langle\chi, e^{-t H} \chi\right\rangle-\right|\langle\Omega, \chi\rangle\right|^{2}|\leq| f(\chi)\right|^{2} e^{-m t}
$$

holds for all $\chi \in \mathcal{D}(f)$ and all $t \geq 0$. It then follows that 0 is a simple eigenvalue of $H$, and that a mass gap of magnitude $m$ exists.

Cluster expansions for field theory were developed and applied in the early 1970 's. ${ }^{19,20}$ This method was originally developed for the two-dimensional examples 
with vectors $\chi$ having the form $\chi=A \Omega$, where $A$ denotes a monomial functions of spatially-averaged, time-zero fields and of heat kernels $e^{-s_{j} H}$, for $s_{j} \geq 0$. Denote this set of $A$ 's by $\mathfrak{A}$. Finite linear combinations of vectors $\chi=A \Omega, A \in \mathfrak{A}$, are dense in $\mathcal{H}$. If we insert vectors $\chi=A \Omega$ into the expectation (11), we obtain a straightforward, functional integral representation. The functional $f(\chi)$ is a norm on $\mathfrak{A}$, with the property $f(A \Omega)=\|A\|_{\mathfrak{a}}=\left\|A^{*}\right\|_{\mathfrak{a}}$; it is defined through a complicated inductively defined construction. We show that for $A \in \mathfrak{A}$, there exists a constant $m>0$, such that for $t \geq 0$,

$$
\left.\left|\left\langle\Omega, A^{*} e^{-t H} A \Omega\right\rangle-\right|\langle\Omega, A \Omega\rangle\right|^{2} \mid \leq\|A\|_{\mathfrak{a}}^{2} e^{-m t} .
$$

The consequence of these bounds is the proof of the existence of a mass gap, uniform for equations with the non-linear interaction restricted to a bounded domain. They also led to the proof of existence and certain regularity properties of the infinite volume limit.

The cluster expansion methods generate a convergent expansion of expectations of fields, for a sufficiently small, strictly-positive coupling constant $\lambda$. Consider an expectation in a $\varphi^{4}$-theory of a product of fields localized in a space-time region $\mathcal{O}^{\prime}$, and with the $\varphi^{4}$-interaction localized in region $\mathcal{O}$ containing $\mathcal{O}^{\prime}$. One performs the expansion for fixed $\mathcal{O}$, but uses the form of the resulting terms to compare different $\mathcal{O}$, and to analyze the limit as $\mathcal{O} \nearrow \mathbb{R}^{2}$. The terms given by the expansion are expectations with the $\varphi^{4}$ interaction localized in a region $\mathcal{O}^{\prime \prime}$, where $\mathcal{O}^{\prime} \subset \mathcal{O}^{\prime \prime} \subset \mathcal{O}$. Furthermore, the magnitude of the sum of terms localized in $\mathcal{O}^{\prime \prime}$ is exponentially small in the size of $\mathcal{O}^{\prime \prime}$. This estimate is independent of $\mathcal{O}$, and is sufficiently strong to allow the comparison of different volumes $\mathcal{O}$. We therefore can estimate the convergence of the original expectation to a limit as the volume $\mathcal{O}$ of interaction tends to infinity.

With these methods, one could establish the first non-trivial example of the Wightman axioms. ${ }^{20}$ Viewed differently, this body of work established the mathematical compatibility of quantum field theory with special relativity. While this work only applied in two-dimensional space-time, it marked the crossing of a major set of obstacles barring progress. In this case, it also showed that the nonperturbative treatment of the equations and their renormalization could be understood at least for these examples in terms of perturbation theory.

\section{Three Dimensions}

The extension of existence to three-dimensional Minkowski space-time for $\varphi=$ $\varphi(x, y, t)$ has been studied for the $\varphi^{4}$ equation, but not for polynomial interactions of higher degree. Unlike in two space-time dimensions where the scalar field $\varphi$ is dimensionless, a scalar field in three dimensional space-time has the dimension length $h^{-1 / 2}$. Hence the singularity of the interaction compared with the unperturbed Hamiltonian grows as the degree of the interaction polynomial increases.

The $\varphi_{3}^{4}$ equation, with $\varphi=\varphi(x, y, t)$ has the form

$$
\square \varphi+\varphi+\lambda \varphi^{3}=0, \quad \text { where } \quad \square \varphi=\varphi_{t t}-\varphi_{x x}-\varphi_{y y} .
$$


Here $\varphi^{3}$ requires a special definition, different from the definition in two-dimensional space-time. The basic stability bound, establishes that the Hamiltonian in a bounded volume is bounded from below. This result is difficult to establish in the three-dimensional case. In order to control stability, we developed a technique called phase cell localization. This allowed one to analyze degrees of freedom associated with a given length scale, and to use these estimates inductively, to analyze degrees of freedom associated with twice the length scale. Phase cell localization ideas are related to ideas of Kadanoff and Wilson's renormalization group. While the paper establishing this result was finished in $1973,{ }^{21}$ it resulted from an evolution of methods and ideas over about four years.

There are two physical renormalizations responsible for the difficulty in analyzing the three-dimensional phenomenon. Each corresponds to a mathematical difficulty that required new techniques to overcome. The first renormalization revolves around the Hamiltonian. As in two-dimensions, the normal ordered Hamiltonian - in the case that the interaction is confined to a bounded volume - is a densely defined bilinear form on Fock space. However, unlike two dimensions, this form does not yield a densely defined operator (as the continuity required by the Riesz representation theorem is not valid). The mathematical problem comes down to understanding how to modify the Hamiltonian in order to obtain a dense operator domain. In fact, to obtain a Hamiltonian operator one must add to the normal-ordered Hamiltonian three renormalization terms: a mass-renormalization term (that is quadratic in the time-zero field $\varphi$ and that diverges logarithmically as a function of a momentum cut-off $\kappa$ ), as well as two vacuum energy renormalization terms that are independent of the field $\varphi$. One of the constant terms is linearly divergent in the momentum cut-off $\kappa$, while the second constant term is logarithmically divergent in $\kappa$.

The second problem is associated with renormalization of the Hilbert space, namely renormalization of the state vectors on which the Hamiltonian acts. This arises because the second-order, linearly-divergent, vacuum-energy renormalization constant in the Euclidean action $S_{2}$ for the time interval $[0, t]$ can be written $S_{2}=t E_{2}+\Lambda_{2}+o(1)$, as $t \rightarrow \infty$. It is the case for constants $\alpha_{2}$ and $\beta_{2}$, that $t E_{2} \sim t \alpha_{2} \lambda^{2} \kappa$ is the linearly divergent vacuum energy renormalization of $t H$ as it occurs in the heat kernel $e^{-t H}$, and $\Lambda_{2} \sim \beta_{2} \lambda^{2} \ln \kappa$ is a logarithmically-divergent remainder that is also time-independent. This remainder gives rise to a multiplicative renormalization of each wave function by the constant $e^{-\Lambda_{2} / 2} \sim e^{-\beta_{2} \lambda^{2} \ln \kappa / 2}$. The constant $\Lambda_{2}$ also forces a change of representation of the canonical commutation relations, and the limiting Hamiltonian $(\kappa \rightarrow \infty)$ acts on a Hilbert space carrying a representation of the CCR that is unitarily inequivalent to the representation for free fields, namely the Fock representation.

This is quite different from the two-dimensional theory. The representation of the Heisenberg relations for the solution to the two-dimensional non-linear wave equation, restricted to any bounded, space-time region is locally, unitarily equivalent to the representation for the free fields. ${ }^{9}$ This local equivalence of representations is known as the "locally Fock" property.

The analysis of these two effects in the three-dimensional theory took considerable work. ${ }^{21}$ Once that stability was in hand, the generalization of the cluster 
expansion method for small, positive $\lambda$ followed in the case of three-dimensional space-time, ${ }^{22}$ and it led to the first example of a non-trivial Wightman theory on $\mathbb{M}^{3}$.

\section{Digging Deeper}

The focus of CQFT was and remains not only to establish existence, but also to develop methods aimed at establishing quantitative and qualitative properties of the particular examples. Thus constructive quantum field theorists did not only attempt to justify expected phenomena, but they also aimed at the broader exploration of physics at a fundamental level - consistent with historical precedents of mathematical integrity. This work also led to establishing physical properties of these examples, including many features of their particle spectrum, the description of scattering in these examples, and the qualitative behavior of the examples as a function of the coupling constants. In this section, we mention only a few of the many phenomena that have captured our imaginations in the above field theories, and about which mathematically complete results have been established.

\subsection{Particles and Scattering}

An initial question to answer concerns whether these quantum field have particle states and whether the solutions to the field theory describe scattering of these particles. There are two standard text-book methods to recover scattering data from a field theory: the theory of Lehmann, Symanzik, and Zimmermann using Green's functions to construct $S$-matrix elements, and the alternate approach of Haag and Ruelle, based on a construction of the wave operator in the Hilbert space. Both these methods require as an hypothesis, the existence of an isolated oneparticle mass hyperboloid in the energy-momentum spectrum — or equivalently the existence of an isolated eigenvalue $m>0$ for the mass operator $M$. This requirement of an isolated eigenvalue is more subtle than the existence of a mass gap. It entails both a lower gap and an upper gap in the spectrum of $M$, as well as the existence of the eigenvalue $m$. If 0 and $m>0$ are eigenvalues of $M$, one expects continuous spectrum on the interval $[2 m, \infty)$, and possible additional eigenvalues in the interval $(m, 2 m)$ in the case of an attractive interaction. These eigenvalues can be interpreted as the masses of particles that are bound states of two mass- $m$ particles, with the lowering of the total mass attributed to the binding energy.

In the $\lambda \mathcal{P}(\varphi)_{2}$ examples, with small $0<\lambda$, this picture has been verified. The first step is to prove the existence of an eigenvalue $m$ which one can interpret as the mass of an isolated particle. A refinement of the cluster expansion techniques leads to that result. ${ }^{20}$ Further analysis shows that $\lambda \varphi^{4}$ equations are repulsive and they do not have bound states, ${ }^{23}$ while $\lambda \varphi^{6}$ equations do have a bound state. ${ }^{24}$

\subsection{Phase Transitions and Non-Uniqueness}

A second qualitative phenomenon occurs for the $\lambda \varphi^{4}$ equation with $\lambda$ large, namely non-uniqueness of the infinite volume limit. Associated with this is the nonuniqueness of the Euclidean Green's functions, of the solutions to the equation 
(3), and of the ground state of $H$. In physics, this non-uniqueness is known as the existence of a phase transition or as degeneracy of the ground state. Phase transitions often occur along with the breaking of a symmetry, in this case breaking of the $\varphi \rightarrow-\varphi$ symmetry of the Lagrangian. There are two successful methods to study this phenomenon. One approach is to develop a new cluster expansion that is valid in the region $\lambda \gg 1$. One can change the parameterization of this equation, ${ }^{25}$ by varying the mass implicit in the definition of the constant $c_{\kappa}$ in (6), and thereby show that (3) for $\lambda \gg 1$ is equivalent to an equation

$$
\square \varphi-\varphi+\lambda \varphi^{3}=0,
$$

with a negative linear term and with $0<\lambda \ll 1$. This parameterization illustrates the two semi-classical minima of the polynomial $\frac{1}{4} \lambda \varphi^{4}-\frac{1}{2} \varphi^{2}$. One can analyze the equation by correlation inequalities. ${ }^{26}$ One can then establish a different sort of convergent cluster expansion in the parameter $\lambda .{ }^{27}$ One can analyze the problem by cluster expansions. This expansion shows that one can construct two distinct, clustering ground states $\Omega_{ \pm}$, corresponding to the expectation $\left\langle\Omega_{ \pm}, \varphi \Omega_{ \pm}\right\rangle \sim \pm \lambda^{-1 / 2}$ of $\varphi$ lying near one of the two minima of the quartic interaction energy density. Furthermore, there is a superselection rule so that for bounded $A$ the different vacua do not mix, $\left\langle\Omega_{+}, A \Omega_{-}\right\rangle=0$. Thus one can decompose the theory into individual examples with a unique vacuum. The cluster expansion selects one of these vacuum states by imposing the value of the field on the boundary of a large box, before taking the infinite volume limit.

\subsection{Zero Mass and Twists}

Super-symmetry is an additional algebraic structure in quantum boson-fermion theories. The mathematical introduction of fermions requires the existence of a $\mathbb{Z}_{2}$-grading $\Gamma$, namely a self-adjoint, root of unity on $\mathcal{H}$. The eigenspaces of $\Gamma$ are defined to be bosonic or fermionic states, respectively. The grading acts on linear transformations by $B \rightarrow B^{\Gamma}=\Gamma B \Gamma$, and modulo questions of domain, every linear transformation can be decomposed uniquely as a sum of two parts that are even (bosonic) and odd (fermionic) under this action. Super-symmetry revolves about the existence of a self-adjoint fermionic operator $Q$ that is the square root of $H=Q^{2}$. This charge $Q$ has a geometric interpretation, as one can define the differential given by the graded commutator $d B=[Q, B]_{\Gamma}=Q B-B^{\Gamma} Q$. Such a structure also arises in non-commutative geometry. ${ }^{28}$

Super-symmetric examples have been extensively studied in constructive quantum field theory, especially in 2-dimensional cylindrical space-times. ${ }^{29,30}$ In these and other cases of two-dimensional constructive field theory, one studies interacting Hamiltonians that are perturbations of free, massive, super-symmetric fields. One routinely introduces a mass, since in two dimensions the massless, scalar boson field is singular. ${ }^{1}$ However, very interesting Lagrangians arise for which a family of supersymmetric interactions (with parameter $0 \leq \lambda \leq 1$ ) appear to share a common Lie group of symmetries of $H$, and for which the $\lambda=0$ endpoint is free. However, in these cases, the Lie symmetry and super-symmetry appear incompatible with an unperturbed massive theory. This forces the question of how to deal with the two-dimsional, massless, bosonic interaction within the framework of constructive 
quantum field theory. We have discussed twist fields, namely multivalued fields on cylindrical space-times that allow massless interactions of a two-dimensional sort. ${ }^{31,32,33,34,35}$

Fundamental to the notion of quantum field is the assumption that the abelian group of space and time translations of $S^{1} \times \mathbb{R}$ has a unitary representation on $\mathcal{H}$ generated by the self-adjoint, commuting operators $P$ and $H$, where $P$ is the momentum. This translation group $e^{i x^{\prime} P+i t^{\prime} H}$ implements the space-time translations of fields, so for the bosonic field with components labelled by $i$,

$$
\varphi_{i}\left(x-x^{\prime}, t+t^{\prime}\right)=e^{i x^{\prime} P+i t^{\prime} H} \varphi_{i}(x, t) e^{-i x^{\prime} P-i t^{\prime} H},
$$

while for the fermionic field with components $\psi_{\alpha, i}$,

$$
\psi_{\alpha, i}\left(x-x^{\prime}, t+t^{\prime}\right)=e^{i x^{\prime} P+i t^{\prime} H} \psi_{\alpha, i}(x, t) e^{-i x^{\prime} P-i t^{\prime} H} .
$$

The unitary twist group $e^{i \theta J}$ has a generator $J$ commuting with $H$ and $P$, and satisfies

$$
e^{i \theta J} \varphi_{i}(x, t) e^{-i \theta J}=e^{i \theta \Omega_{i}^{b}} \varphi_{i}(x, t) .
$$

The fermionic time-zero field $\psi_{\alpha, i}$ satisfies

$$
e^{i \theta J} \psi_{\alpha, i}(x, t) e^{-i \theta J}=e^{i \theta \Omega_{\alpha, i}^{f}} \psi_{\alpha, i}(x, t)
$$

The twisting angles $\Omega=\left\{\Omega_{i}^{b}, \Omega_{\alpha, i}^{f}\right\}$ are given constants that characterize the twist generator $J$, up to an additive constant $\hat{c} / 2$, chosen so that $\pm J$ have the same spectrum. Then the zero-particle vector $\Omega_{0} \in \mathcal{H}$ satisfies

$$
J \Omega_{0}=\frac{1}{2} \hat{c} \Omega_{0}, \text { with } \hat{c}=\sum_{i=1}^{n}\left(\Omega_{2, i}^{f}-\Omega_{1, i}^{f}\right) .
$$

A twist quantum field on a circle $S^{1}$ is a field for which these two groups are related. If the circle has length $\ell$, then

$$
\varphi_{i}(x+\ell, t)=e^{i \chi_{i}^{b}} \varphi_{i}(x, t),
$$

and

$$
\psi_{\alpha, i}(x+\ell, t)=e^{i \chi_{\alpha, i}^{f}} \psi_{\alpha, i}(x, t),
$$

for all $x \in S^{1}$ and $t \in \mathbb{R}$. The set of twisting angles $\chi=\left\{\chi_{i}^{b}, \chi_{\alpha, i}^{f}\right\}$ is taken so that no twisting phase equals one. In case the superpotential $V$ satisfies the quasi-homogenity condition

$$
V(z)=\sum_{i=1}^{n} \Omega_{i} z_{i} \frac{\partial}{\partial z_{i}} V(z)
$$

we choose

$$
\left\{\chi_{i}^{b}, \chi_{\alpha, i}^{f}\right\}=\left\{\Omega_{i}^{b} \phi, \Omega_{\alpha, i}^{f} \phi\right\}
$$




\subsection{Twists Break Super-symmetry}

The twist fields act in many ways like massive fields, and in fact the Fourier momenta are shifted from zero by the amount $\chi / \ell$, choosing the $\chi$ appropriate for each component. Thus twist fields are not infra-red singular at $k=0$ like periodic fields, and twist fields can yield translation-invariant, twist-invariant Hamiltonians. However, twist fields are not totally compatible with super-symmetry. On the other hand, one finds that for a complex interaction where one expects $N=2$ supersymmetry, one can preserve half the supercharges. Furthermore, one can estimate the errors in the full super-symmetry algebra. One expect in a periodic example that there are self-adjoint charges $Q_{1}$ and $Q_{2}$ such that

$$
Q_{1}^{2}=H+P, \quad \text { and } \quad Q_{2}^{2}=H-P .
$$

In fact, we come closest to this situation when $H$ has the form

$$
H=H_{0}+\int_{0}^{\ell} H_{I}(x) d x,
$$

where

$$
\begin{aligned}
H_{I}(x)= & \sum_{j=1}^{n}\left|V_{j}(\varphi(x))\right|^{2} \\
& +\sum_{i, j=1}^{n} \psi_{i, 1}(x) \psi_{j, 2}(x)^{*} V_{i j}(\varphi(x)) \\
& +\sum_{i, j=1}^{n} \psi_{i, 2}(x) \psi_{j, 1}(x)^{*} V_{i j}(\varphi(x))^{*} .
\end{aligned}
$$

Then there is an operator $Q_{1}$ invariant under translations and twists, and an operator $Q_{2}$ such that

$$
Q_{1}^{2}=H+P, \quad \text { and } \quad Q_{2}^{2}=H-P+\phi \mathcal{R} .
$$

Here $\mathcal{R}$ is an operator independent of $\lambda$, and satisfies for some constants $0<\epsilon$,

$$
\pm \epsilon \mathcal{R} \leq H+I \text {. }
$$

Such an estimate allows us to define and study the twisted partition function

$$
\mathfrak{Z}^{V}=\operatorname{Tr}_{\mathcal{H}}\left(\Gamma e^{-i \theta J-i \sigma P-\beta H}\right) .
$$

Amazingly, this partition function $\mathfrak{Z}^{V}$, which is a geometric invariant, ${ }^{28,36,37}$ can be computed. It displays a hidden modular symmetry, and it can be expressed in terms of elementary theta functions. In fact $\mathfrak{Z}^{V}$ depends on $V$ only through its universality class determined by the numbers $\left\{\Omega_{i}\right\} .{ }^{33}$ In terms of $\tau=(\sigma+i \beta) / \ell$

$$
\mathfrak{Z}^{V}(\tau, \theta, \phi)=z^{\hat{c} / 2} \prod_{i=i}^{n} \frac{\vartheta_{1}\left(\tau,\left(1-\Omega_{i}\right)(\theta-\phi \tau)\right)}{\vartheta_{1}\left(\tau, \Omega_{i}(\theta-\phi \tau)\right)}
$$




\section{For the Millennium: Gauge Theory in Four Dimensions}

Relations between field theory and geometry also arise both in gauge theories. The field $F$ is the curvature of a connection $A$, and the classical equations for $F$ transform covariantly under a change of coordinates (change of gauge). Classical Yang-Mills fields take values in the Lie algebra of the gauge group. Despite the farreaching success of constructive quantum field theory, the original puzzle explained in $\S 1$ remains unresolved. Can one find a non-trivial, non-linear quantum field in four-dimensional space-time?

The most promising candidate for a non-trivial and physically-interesting field theory on Minkowski 4-space is the Yang-Mills theory with an $S U(2)$ gauge group. The Yang-Mills field $F$ is defined in terms of a Lie-algebra valued connection $A$,

$$
F=d A+A \wedge A .
$$

The Euclidean Yang-Mills Lagrangian is $\|F\|^{2}$, where the squared norm includes a trace over $S U(2)$ and an integral over space-time. Perturbation theory involves the study of the interaction in powers of the non-linearity arising from $A \wedge A$, and it indicates that this Yang-Mills example is asymptotically free. The physical interaction becomes weaker at high energy, and for this reason, the objections from perturbation theory suggesting the triviality of $\varphi_{4}^{4}$ do not carry over to Yang-Mills interactions in four-space. Furthermore, physicists expect that this example will have a mass gap. Therefore one can pose the following:

Problem. Prove the existence of a quantum field theory on $\mathbb{M}^{4}$ satisfying the Euclidean axioms for gauge theories, agreeing with $S U(2)$-Yang-Mills physics texts in lowest order perturbation theory in $g$, and possessing a gap in the mass spectrum.

This problem has been attacked by a number of people, leading to substantial partial progress. ${ }^{38,39}$ One can envision the positive future answer to the question of the existence of an asymptotically-free, four-dimensional gauge theory on a cylindrical space-time, although the infra-red (infinite-volume) limit still seems beyond grasp. The non-abelian nature of the gauge group means that the curvature $F=d A+A \wedge A$ will lead to a quartic term in the energy. Such a potential might, in fact, rise rapidly enough to confine the wave-function. Feynman, toward the end of his life believed that he understood this.

\section{Acknowledgments}

This work was written in part at the Clay Mathematics Institute. The author acknowledges support in part by the grant DE-FG02-94ER-25228 from the Department of Energy.

\section{References}

1. Arthur Wightman, Introduction to some aspects of the relativistic dynamics of quantized fields, in High Energy Electromagnetic Interactions and Field Theory, Maurice Lévy, Ed., Gordon and Breach, London, (1967). 
2. Arthur Jaffe, Existence Theorems for a Cut-off $\lambda \varphi^{4}$ Field Theory, in Conference on the Mathematical Theory of Elementary Particles, R. Goodman and I. Segal, Eds., MIT Press, Cambridge, Massachusetts 1966.

3. Oscar Lanford, Construction of quantum fields interacting by a cutoff Yukawa coupling, Princeton thesis 1966.

4. Kurt Symanzik, A modified model of Euclidean quantum field theory, Courant Institute of Mathematical Sciences Report IMM-NYU 327, June 1964.

5. Kurt Symanzik, Euclidean Quantum Field Theory, in Local Quantum Theory, pp. 152-226, R. Jost, Editor, Academic Press, New York 1969.

6. Edward Nelson, A quadratic interaction in two dimensions, in Conference on the Mathematical Theory of Elementary Particles, R. Goodman and I. Segal, Eds., MIT Press, Cambridge, Massachusetts 1966.

7. James Glimm and Arthur Jaffe, A $\lambda \varphi^{4}$ quantum field theory without cut-offs, I, Phys. Rev. 176, 1945-1961 (1968).

8. James Glimm and Arthur Jaffe, The $\lambda \varphi_{2}^{4}$ quantum field theory without cutoffs, II. The field operators and the approximate vacuum, Ann. of Math. 91, 362-401 (1970).

9. James Glimm and Arthur Jaffe, The $\lambda \varphi_{2}^{4}$ quantum field theory without cut-offs, III. The physical vacuum, Acta Math. 125, 203-267 (1970).

10. James Glimm and Arthur Jaffe, The $\lambda \varphi_{2}^{4}$ quantum field theory without cutoffs, IV. Perturbations of the Hamiltonian, Jour. Math. Phys. 13, 1568-1584 (1972).

11. James Glimm and Arthur Jaffe, Quantum Physics, Springer, New York 1987.

12. John Cannon and Arthur Jaffe, Lorentz covariance of the $\lambda \phi_{2}^{4}$ quantum field theory, Commun. Math. Phys. 17, 261-321 (1970).

13. Edward Nelson, Quantum fields and Markoff fields, Proc. Sympos. Pure Math. XXIII 1971, pp. 413-420, Amer. Math. Soc., Providence, R.I. 1973.

14. Francesco Guerra, Uniqueness of the vacuum energy density and van Hove phenomenon in the infinite volume limit for two-dimensional self-coupled Bose fields, Phys. Rev. Lett. 28 (1972), 1213-1215.

15. Francesco Guerra, Lon Rosen, and Barry Simon, The $\mathcal{P}(\varphi)_{2}$ Euclidean quantum field theory as classical statistical mechanics, Ann. Math. 101, 111-259.

16. Konrad Osterwalder and Robert Schrader, Axioms for Euclidean Green's functions, Commun. Math. Phys. 31 (1973), 83-112, and Commun. Math. Phys. 42 (1975), 281-305.

17. James Glimm and Arthur Jaffe, A note on reflection positivity, Lett. Math. Phys. 3 (1979), 377-378.

18. Konrad Osterwalder and Robert Schrader, Euclidean Fermi fields and a Feynman-Kac formula for boson-fermion interactions, Helv. Phys. Acta 46 (1973), 227-302.

19. James Glimm, Arthur Jaffe, and Thomas Spencer, The particle structure of the weakly coupled $P(\phi)_{2}$ model and other applications of high temperature expansions, Part II: The Cluster Expansion, in Constructive Quantum Field Theory, A.S. Wightman (ed.), Springer Lecture Notes in Physics Volume 25, Springer, Berlin, New York (1973).

20. James Glimm, Arthur Jaffe, and Thomas Spencer, The Wightman axioms and 
particle structure in the weakly coupled $P(\varphi)_{2}$ quantum field model, Ann. of Math. 100 (1974), 585-632.

21. James Glimm and Arthur Jaffe, Positivity of the $\varphi_{3}^{4}$ Hamiltonian, Fortschr. Phys. 21 (1973), 327-376. Reprinted in Constructive Quantum Field Theory, Selected Papers, Birkhäuser Boston 1985.

22. Joel Feldman and Konrad Osterwalder, The Wightman axioms and the mass gap for weakly coupled $\varphi_{3}^{4}$ quantum field theories, Ann. Physics 97 (1976), 80-135.

23. Thomas Spencer and Francesco Zirilli, Scattering states and bound states in $\lambda \mathcal{P}(\varphi)_{2}$, Commun. Math. Phys. 49 (1976), 1-16.

24. Jon Dimock and Jean-Pierre Eckmann, On the bound state in weakly coupled $\lambda\left(\varphi^{6}-\varphi^{4}\right)_{2}$, Commun. Math. Phys. 51 (1976) 41-54.

25. James Glimm, Arthur Jaffe, and Thomas Spencer, Existence of phase transitions for $\phi_{2}^{4}$ quantum fields, Mathematical Methods of Quantum Field Theory, F. Guerra, D. Robinson, and R. Stora, Eds., CNRS, Paris 1976.

26. James Glimm, Arthur Jaffe, and Thomas Spencer, Phase transitions for $\varphi_{2}^{4}$ fields, Commun. Math. Phys. 45 (1975) 203-216.

27. James Glimm, Arthur Jaffe, and Thomas Spencer, A convergent expansion about mean field theory, I. Ann. Phys. 101 (1976), 610-630, and II. Ann. Phys. 101 (1976), 631-669.

28. Alain Connes, Noncommutative differential geometry, Publ. Mathematiques IHES 62 (1986), 257-360.

29. Arthur Jaffe, Andrzej Lesniewski, and Jonathan Weitsman (1988) The twodimensional, $\mathrm{N}=2$ Wess-Zumino model on a cylinder, Commun. Math. Phys. $114147-165$.

30. Arthur Jaffe and Andrzej Lesniewski (1988) A priori estimates for the $\mathrm{N}=2$, Wess-Zumino model on a cylinder, Commun. Math. Phys. 114, 553-575.

31. Arthur Jaffe, Twist positivity, Ann. Phys. 278 (1999), 10-61.

32. Olivier Grandjean and Arthur Jaffe, Twist fields and constructive quantum field theory, J. Math. Phys., to appear.

33. Arthur Jaffe, The elliptic genus and hidden symmetry, Commun. Math. Phys., to appear.

34. Arthur Jaffe, Twist fields, the elliptic genus, and hidden symmetry, Proc. Nat. Acad. Sci., 97 (2000), 1418-1422.

35. Arthur Jaffe, Twist fields and constructive quantum field theory, in preparation.

36. Arthur Jaffe, The holonomy expansion, index theory, and approximate supersymmetry, Ann. Phys. 279 (2000), 161-262.

37. Arthur Jaffe, Quantum harmonic analysis and geometric invariants, Advances in Math. 143, 1-110 (1999).

38. Tadeusz Balaban, Renormalization group approach to lattice gauge field theories. I. Generation of effective actions in a small field approximation and a coupling constant renormalization in 4D, Commun. Math. Phys. 109 (1987), 249-301.

39. Jacques Magnen, Vincent Rivasseau, and Roland Sénéor, Construction of $Y M_{4}$ with an infrared cutoff, Commun. Math. Phys. 155 (1993), 325-383. 